\title{
BMJ Open Neighbourhood environment, physical activity, quality of life and depressive symptoms in Hong Kong older adults: a protocol for an observational study
}

\author{
Ester Cerin, ${ }^{1,2}$ Cindy H P Sit, ${ }^{3}$ Casper J P Zhang, ${ }^{1}$ Anthony Barnett, ${ }^{2,4}$ \\ Martin M C Cheung, ${ }^{5}$ Poh-chin Lai, ${ }^{6}$ Janice M Johnston, ${ }^{1}$ Ruby S Y Lee ${ }^{5}$
}

To cite: Cerin E, Sit CHP, Zhang CJP, et al.

Neighbourhood environment, physical activity, quality of life and depressive symptoms in Hong Kong older adults: a protocol for an observational study. BMJ Open 2016;6:e010384. doi:10.1136/bmjopen-2015010384

- Prepublication history for this paper is available online. To view these files please visit the journal online (http://dx.doi.org/10.1136/ bmjopen-2015-010384).

Received 28 October 2015 Revised 9 December 2015 Accepted 10 December 2015

CrossMark

For numbered affiliations see end of article.

Correspondence to Professor Ester Cerin; ecerin@hku.hk

\section{ABSTRACT}

Introduction: The neighbourhood environment can assist the adoption and maintenance of an active lifestyle and affect the physical and mental well-being of older adults. The psychosocial and behavioural mechanisms through which the environment may affect physical and mental well-being are currently poorly understood.

Aim: This observational study aims to examine associations between the physical and social neighbourhood environments, physical activity, quality of life and depressive symptoms in Chinese Hong Kong older adults.

Methods and analyses: An observational study of the associations of measures of the physical and social neighbourhood environment, and psychosocial factors, with physical activity, quality of life and depressive symptoms in 900 Hong Kong older adults aged 65+ years is being conducted in 2012-2016. The study involves two assessments taken 6 months apart. Neighbourhood walkability and access to destinations are objectively measured using Geographic Information Systems and environmental audits. Demographics, socioeconomic status, walking for different purposes, perceived neighbourhood and home environments, psychosocial factors, health status, social networks, depressive symptoms and quality of life are being assessed using validated interviewer-administered selfreport measures and medical records. Physical functionality is being assessed using the Short Physical Performance Battery. Physical activity and sedentary behaviours are also being objectively measured in approximately $45 \%$ of participants using accelerometers over a week. Physical activity, sedentary behaviours, quality of life and depressive symptoms are being assessed twice (6 months apart) to examine seasonality effects on behaviours and their associations with quality of life and depressive symptoms.

Ethics and dissemination: The study received ethical approval from the University of Hong Kong Human Research Ethics Committee for Non-Clinical Faculties (EA270211) and the Department of Health (Hong Kong SAR). Data are stored in a passwordprotected secure database for 10 years, accessible only
Strengths and limitations of this study

- The use of objective and self-reported data on the neighbourhood environment and physical activity will enable a better understanding of potential environmental and behavioural influences on quality of life and depressive symptoms.

- The recruitment of participants from neighbourhoods stratified by socioeconomic status and walkability will maximise the variability of environmental exposures and behavioural outcomes enabling a more accurate estimation of doseresponse relationships.

- This is an observational study with a 6-month follow-up assessment to examine seasonality effects on physical activity and their associations with changes in quality of life and depressive symptoms. Hence, causal relations cannot be inferred.

to the named researchers. Findings will be submitted for publication in peer-reviewed journals.

\section{INTRODUCTION}

There is compelling evidence that engagement in regular moderate-to-vigorous intensity physical activity (PA) can lower the risk of all-cause mortality, a range of chronic diseases, dementia and depression, and contribute to independence and social well-being in older adults. ${ }^{1-4}$ Additionally, recent studies indicate that, in this age group, some health benefits may even occur from light-intensity activities $^{5}$ including easy walking $(<4.5 \mathrm{~km} / \mathrm{h})$ and reduction in sedentary activities. ${ }^{6}$ In general, the accrual of at least $30 \mathrm{~min}$ of low-to-moderate ${ }^{2}{ }^{5} \mathrm{PA}$ on 5 or more days of the week is deemed necessary for health benefits. ${ }^{2}$ PA prevalence studies indicate that a 
large percentage of a general population of older adults in Western as well as Asian countries, including Hong Kong, ${ }^{7}$ does not meet these guidelines. ${ }^{8}$ It is thus important to examine the determinants of PA participation and sedentary behaviour (SB) specific to older adults so that age-appropriate interventions can be designed.

In recognition that environment can impact on PA levels and SB, over the past 15 years researchers have gathered substantial supporting evidence on the influence of the neighbourhood environment on adults' PA and SB. ${ }^{9}{ }^{10}$ An increasingly growing number of studies have examined these associations in older adults (aged $65+$ years). ${ }^{11-14}$ This area of research is particularly important because older adults are likely to be most influenced by the physical features of the environment since mobility, independence and social contacts can be greatly limited by a poorly designed community. The provision of enabling and supportive environments is one of the three main priorities outlined by the Madrid International Plan of Action on Aging ${ }^{15}$ on which international efforts are to be focused in respect to population ageing.

Studies on older adults have found significant positive relationships of traffic/pedestrian safety (crosswalks; curb cuts; safe footpaths), access to commercial services, and access to recreational facilities/places with PA, especially walking, ${ }^{11-14}{ }^{16}$ and opposite relationships with SB. ${ }^{17}$ There is some evidence that these relationships might depend on other physical and social environmental characteristics ${ }^{16-19}$ and on individual attributes, such as physical health status, ${ }^{20}$ age, ${ }^{11}{ }^{21}$ socioeconomic status, ${ }^{22} 23$ employment ${ }^{24}$ and attitude towards walking. ${ }^{25}$ The detection of interacting, multilevel effects of individual, social and environmental factors on $\mathrm{PA}$ and $\mathrm{SB}$ behaviour is in line with social ecological models of health behaviour, representing the main theoretical approach of studies on the environment-PA and environment-health relationships. ${ }^{26}$ Yet, how individual, social and environmental factors act and interact to facilitate or hinder PA and SB of older adults is largely unknown. ${ }^{10} 172022 \quad 27$ Multilevel, multifactorial studies sufficiently powered to examine a vast array of individual, social and environmental factors are needed.

Apart from affecting residents' $\mathrm{PA}$ and SB, physical and social neighbourhood environments have been linked to quality of life (QoL) and well-being indicators in older adults. ${ }^{18}$ Specifically, neighbourhood walkability, operationalised as land-use mix, street connectivity, number of public transit stations and amount of green and open space, was found to be associated with a lower risk of cardiovascular disease. ${ }^{28} 29$ Traffic safety and presence of parks were predictive of better mental and physical well-being. ${ }^{30-32}$ The observed associations are likely to be partly due to the effect of these specific environmental attributes on PA, which in turn contributes to better mental and physical health. ${ }^{33}$ However, it is also plausible to assume that some of the observed effects of the environment on mental well-being in particular might be attributable to greater levels of neighbourhood satisfaction and opportunities to engage in social activities independent of PA. ${ }^{34}{ }^{35}$ Yet, no studies have thus far comprehensively examined potential pathways of influence of neighbourhood environment characteristics on mental well-being (eg, depressive symptoms) in older adults. While there is some evidence that walkable neighbourhoods are related to higher levels of mental well-being, ${ }^{30}{ }^{31}$ neighbourhood satisfaction, ${ }^{36}$ social cohesion $^{37}$ and social activities, ${ }^{34} 38$ the extent to which the physical environment-mental well-being relationships are explained by PA, access to social networks and neighbourhood satisfaction in older adults is unknown. It is also unknown how aspects of the neighbourhood environment may impact on physical well-being. For example, while mixed-use neighbourhoods are conducive to walking, they are often associated with high levels of noise and traffic. Noise pollution and high traffic load may increase stress ${ }^{39}$ and interfere with sleep, ${ }^{40}$ and cancel out the physical benefits of engaging in regular walking for utilitarian purposes. These are still unanswered important research questions.

Although the number of studies on environmental correlates of PA, SB and health in older adults is growing, the great majority of them were conducted in Western, relatively low-density urban areas. ${ }^{13}$ Asian ultradense metropolises present unique environmental characteristics (eg, crowdedness, air and noise pollution, an efficient public transport network, indoor places for walking $)^{4142}$ that may directly or interactively affect PA, SB and well-being of older adults in yet undocumented ways. Patterns of environment-health and environment$\mathrm{PA} / \mathrm{SB}$ relationships observed in Western locations are unlikely to be entirely generalisable to Hong Kong also due to cross-cultural differences in social network types, ${ }^{43}$ attitudes towards specific forms of activities, ${ }^{7}$ policy and other culture-dependent factors. Although recent studies have reported findings on associations of the neighbourhood environment with various forms of PA and sitting time in older Chinese dwellers in Hong Kong, ${ }^{11}{ }^{12}{ }^{16-18}$ these studies focused on perfectly mobile and healthy individuals. Thus, it is not known whether the observed patterns of associations hold for a more general population of Hong Kong community-dwelling older adults. Also, no studies have looked at the potential effects of the neighbourhood environment on QoL and mental well-being in this population. Therefore, it is necessary to examine environment-PA/SB, environment-QoL and environmentmental well-being associations as well as to identify the complex nature of the individual, social and environmental influences among Chinese older adults in Hong Kong.

Finally, the preponderance of studies on environment-PA/SB in older adults adopted a cross-sectional design, not allowing the identification of environmental predictors of changes in $\mathrm{PA}$ and $\mathrm{SB}$, and the estimation of related seasonal effects. ${ }^{13} 1744$ As it is important to 
identify how changes in weather conditions can affect changes in PA/SB ${ }^{44} 45$ and health-related QoL, ${ }^{46}$ especially in an environment with a tropical, humid climate such as that of Hong Kong, ${ }^{47-49}$ this study proposes to collect outcome measures (PA/SB, QoL and depressive symptoms) twice, 6 months apart, so that seasonal effects can be studied and associations of short-term changes in PA, SB and other outcome variables can be examined.

To address the knowledge gaps identified above, the Active Lifestyle and the Environment in Chinese Seniors (ALECS) study seeks to: (1) assess the independent and interactive effects (associations) of aspects of the social and physical neighbourhood environment, individual characteristics (sociodemographics and health status) and psychosocial (social support and networks) factors on PA, SB, QoL and depressive symptoms in Chinese older adults in Hong Kong (aged 65+ years); (2) examine the mediating effects of PA, SB and accessibility of social networks on the relationships between neighbourhood environment characteristics and depressive symptoms; (3) examine potential psychosocial mechanisms explaining the relationships of neighbourhood environment characteristics with $\mathrm{PA}$ and $\mathrm{SB}$; and (4) examine associations of changes in PA, SB, QoL and depressive symptoms and neighbourhood environment characteristics and psychosocial factors as moderators of such associations. Figure 1 provides a simplified diagram of the patterns of relationships that will be studied.

\section{METHODS AND ANALYSIS}

All applicable elements of the standardised checklist for the Strengthening the Reporting of Observational Studies in Epidemiology (STROBE) recommendations were used to ensure that all important aspects relevant to study design and data collection were addressed. ${ }^{50}$

\section{Design}

The ALECS study is an observational, primarily crosssectional study of the associations of aspects of the physical and social neighbourhood environment, and psychosocial factors, with PA, QoL and depressive symptoms in 900 Hong Kong older adults aged 65+ years living in preselected Hong Kong neighbourhoods. It also includes a longitudinal component aimed at capturing potential seasonal changes in PA, QoL and depressive symptoms, their associations, and the moderating effects of baseline environmental and psychosocial factors on these associations.

Sociodemographic information, health status, neighbourhood satisfaction, psychosocial indicators, perceived and objectively assessed neighbourhood environment, self-reported PA and sitting time, body mass index (BMI), QoL and depressive symptoms are being measured in the full sample. In approximately $45 \%$ of the sample, PA and sedentary time are being assessed using accelerometers (activity monitors) for seven consecutive days. Data are being collected from late 2012 to early 2016 .

\section{Neighbourhood and participant selection \\ Neighbourhood selection}

This study adopts a two-stage stratified sampling strategy designed to allow comparisons between older adults living in neighbourhoods stratified on the basis of their transport-related 'walkability' characteristics and median household income (representing neighbourhood-level socioeconomic status). This design was chosen because it creates the greatest range in the primary exposure variables (neighbourhood physical environment) related to walkability, while allowing to control for neighbourhood-level socioeconomic differences. ${ }^{22}$ Neighbourhood-level socioeconomic status is also included in the design because PA varies by income, and studies indicate that walkability interacts with neighbourhood income in its association with PA in adults ${ }^{51}$ and older adults. ${ }^{14}$

The selection of neighbourhoods is based on a multidimensional index to represent the 'walkability' of Tertiary Planning Units (TPUs). TPUs are the most appropriate geographical scale for the selection of neighbourhoods in Hong Kong as they are linked with Census sociodemographic data, such as median household income, median age and education. ${ }^{42}$ For each TPU, the walkability index is derived as a function of net residential density, land use mix and intersection density. ${ }^{11} 52$ Walkability variables were selected on the basis of previous studies and are computed from Census, land use data and street centreline files. ${ }^{53}$ Net residential density is calculated by dividing the number of dwellings in a TPU (Census data) by its net residential land area (data provided by the Lands Department of Hong Kong). Land use mix is being computed using information on the area of five land use categories (residential, commercial, industrial, institutional, open space/park provided by the Lands Department, Hong Kong) in an entropy equation. ${ }^{53}$ Intersection density is calculated by dividing the number of three-or-more-way intersections by the TPU area in square kilometres. Intersections are extracted from street network data sets (centreline data) provided by the authorities. The raw scores for these three components of transport-related walkability are normalised (converted to z-scores) and summed to create a walkability index. The walkability index and related components have been found to be associated with walking in Western ${ }^{54}$ and Chinese older adults in Hong Kong. ${ }^{16} 55$

TPUs are ranked and divided into deciles on the basis of the walkability index. The top four and bottom four deciles are labelled 'high walkability' and 'low walkability,' respectively. Similarly, the median household income data for each TPU is deciled. The bottom four deciles constitute the 'low income' category, and the top four deciles make up the 'high income' category. Thirty candidate TPUs within each of the four Walkability by 
Figure 1 Simplified diagrammatic representation of study aims.

Study aim 1

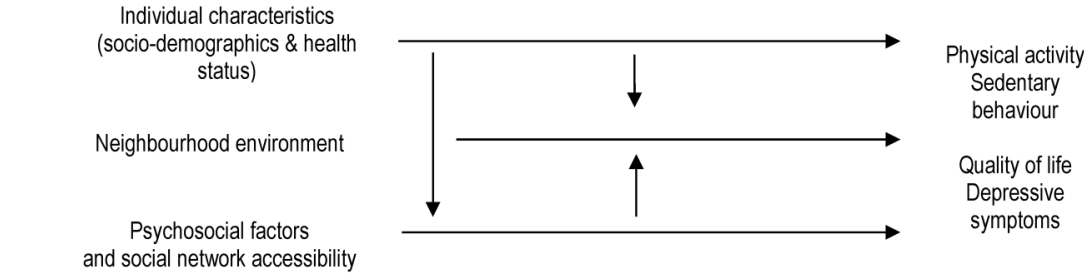

Study aim 2

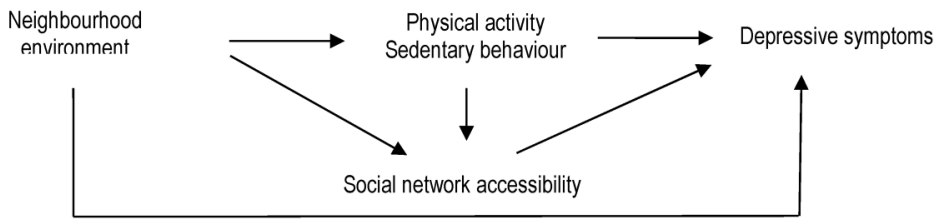

Study aim 3

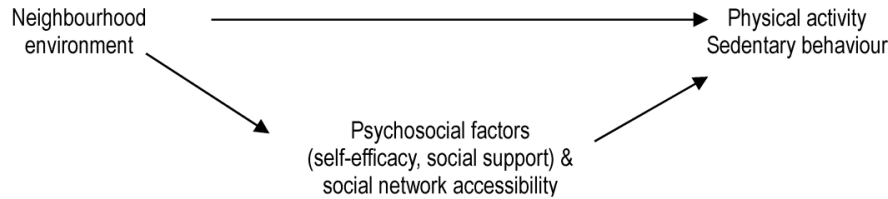

Study aim 4

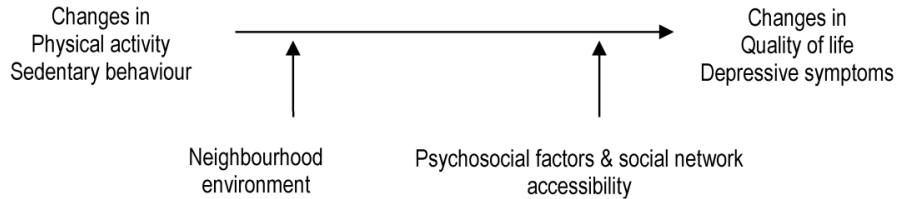

socioeconomic status categories (high walkable/high socioeconomic status; high walkable/low socioeconomic status; low walkable/high socioeconomic status; low walkable/low socioeconomic status) are identified and selected by investigators after in-person visits. Hence, participants are recruited from a total of 120 TPUs (average of 7-8 participants per TPU).

\section{Participant selection and recruitment}

Owing to the inability to directly obtain residential address and other contact data (phone numbers) of potentially eligible participants in Hong Kong associated with the Personal Data (Privacy) Ordinance, ${ }^{56}$ participants are being recruited in person from the Elderly Health Centres (EHC) of the Department of Health, Hong Kong SAR (two-thirds of the sample) and from elderly community centres (a third of the sample). The EHCs (18 distributed across the whole Hong Kong territory) were established by the Department of Health of the Government of Hong Kong SAR to provide comprehensive primary care services for Hong Kong residents aged 65 years and over. Participants are recruited through the EHCs because of their appropriate age, ability to pre-screen their health with the assistance of the EHC staff, their representativeness of the general elderly population of Hong Kong in terms of health and age, and their observed willingness to participate in health-related studies endorsed by the EHCs. ${ }^{57}$ The response rate in a previous study on EHC members aimed at the development and validation of measures of perceived environment and walking for different purposes for the Hong Kong elderly population was $78 \%{ }^{11}$ On the basis of previous studies and the team's experience, if we were to recruit older adults directly from residential addresses, the expected response rate would be lower than $10 \%$. Such a recruitment procedure would be more costly and result in a biased sample (overrepresentation of seniors living with family members and with higher educational attainment) and lower quality data. Yet, to examine possible biases in outcome variables associated with recruiting participants through the EHCs versus the general community, a third of the sample is recruited from the general elderly community centres with no formal provision of medical and health services.

EHC and elderly community centre selection is based on convenience and proximity to large numbers of TPUs in each of the four neighbourhood types. Within 
each EHC and community centre, eligible participants attending the centre during research staff daily visits to the centre are invited to participate in the study by research staff. Inclusion criteria are: (1) being 65 years of age or older; (2) being Cantonese speaking and able to communicate verbally; (3) having lived in one of the selected TPUs for at least 6 months; (4) being able to walk unassisted for at least $10 \mathrm{~m}$; (5) being cognitively intact (Mini-Mental State Examination score $>22^{58}$ ). We are aiming to get an age and sex distribution proportional to the percentages observed in the population (65-74 years: $60 \%$; $75-84$ years: $30 \%$; $85+$ years: $10 \%$; females approximately 60\%) and a balanced number of participants by neighbourhood type. A subsample of approximately 100 participants is randomly selected from each neighbourhood type to participate in the objective assessment of PA and sedentary time component.

\section{Sample size}

In adjusted multilevel models, it has been estimated that a sample of 900 older adults recruited from 30 TPUs within each stratum (120 in total) would allow the detection of a small effect size (ie, $<1.0 \%$ of the explained outcome variance) with $80 \%$ power assuming a TPU-level clustering effect (Intraclass Correlation Coefficient) of $<5 \%$, as observed in a previous similar study on Hong Kong older adults. ${ }^{11}$ The calculated sample size assumes a two-tailed probability level of $5 \%$, and a regression model with 12 covariates explaining $25 \%$ of the outcome variance (comparable to what might be expected from the selected variables). The corresponding effect size detectable in a subsample of approximately 400 participants with objectively assessed PA and sedentary time data is approximately $2.5 \%$ of the explained outcome variance (representing a small effect size).

\section{Exposures, outcomes and covariates}

Table 1 reports all the exposures, outcomes and covariates that are being assessed in this study.

\section{Exposures \\ Geographic Information Systems (GIS) measures of the neighbourhood environment}

GIS data provide spatially referenced information that can be employed to create objective measures of the physical and social neighbourhood environment. They are used to objectively characterise the neighbourhood environment of study participants defined as road network buffers within a certain distance from home (eg, $400 \mathrm{~m}$ or $1 \mathrm{~km}$ ). Road network buffers can be created to define areas that can be reached via the street network but exclude inaccessible portions of land due to major barriers (river, sea or railway). This study uses two main buffer sizes to define participants' neighbourhoods: $400 \mathrm{~m}$ and $1 \mathrm{~km}$ street-network buffers. Although most studies on the environment and PA have used
$1 \mathrm{~km}$ street-network buffers, ${ }^{59}$ there is some evidence that smaller areas ( $400 \mathrm{~m}$ buffers) may be appropriate to characterise the neighbourhood in high-density environments. ${ }^{11} 30$ This study aims to compare these two different geographical scales in the context of Hong Kong. A list of GIS measures of the neighbourhood environment is given in table 1 .

Following a protocol developed for the International Physical Activity and the Environment (IPEN)-Adults study, a multicountry study on environment and PA of which Hong Kong was a study site, ${ }^{59}{ }^{60}$ a walkability index is computed for each participant and buffer size. The walkability index represents the sum of the following standardised variables: net dwelling density, intersection density and land use mix (defined as described above). GIS is also employed to define the percentage of older adult $(65+$ years) population in the neighbourhood based on Census data. This represents a neighbourhood social environment feature that has been found to be predictive of walking in older adults and may facilitate social interaction. ${ }^{61}$ GIS is also being used to quantify access to recreational facilities and parks. This includes density of recreational facilities (number of such destinations per $\mathrm{km}^{2}$ ), number of parks contained and intersecting a specific buffer, street-network distance to the nearest recreational facility and streetnetwork distance to the nearest park. These features have been linked with higher levels of $\mathrm{PA}^{19}$ and better mental health. ${ }^{30}$ GIS-based access to transit points is defined as the street-network distance to the nearest transit stop (bus, railway, tram or subway) and as transit point density (number of transit points per $\mathrm{km}^{2}$ ), which have been found to be predictive of higher levels of overall utilitarian walking $^{55}$ and lower levels of self-reported sitting in Hong Kong healthy and mobile older adults. ${ }^{17}$

\section{Environmental audit measures of the neighbourhood environment}

Extant GIS data on environmental characteristics are limited and sometimes outdated or inaccurate. Thus, it is desirable to complement GIS information with data collected using on-site environmental audits (systematic observation by trained raters). The Environment in Asia Scan Tool-Hong Kong version (EAST-HK), a validated audit tool specifically developed for use in Hong Kong, ${ }^{62}$ and the Public Open Space Tool (POST), a validated instrument to assess feature of parks and public open spaces, ${ }^{63}$ are employed to measure environmental features within $400 \mathrm{~m}$ road network buffers surrounding the participants' homes. The POST assesses features of public open spaces including types of activities, amenities, environmental quality and safety. ${ }^{63}$ The EAST-HK quantifies features of the pedestrian environments along street segments, including traffic safety, pedestrian infrastructure, personal safety, aesthetics and access to various types of destinations and services. Aspects of personal safety include the following social environment characteristics: presence of people of different ages, presence of people arguing/fighting, presence of prostitutes and homeless people, other signs of social disorder). 
Table 1 Summary of study exposures, outcomes, covariates, moderators and/or mediators

\begin{tabular}{|c|c|c|c|}
\hline Exposures & Assessment & Outcome measures & Assessment \\
\hline \multicolumn{2}{|l|}{$\begin{array}{l}\text { Neighbourhood environment } \\
\text { (method of data collection) }\end{array}$} & \multicolumn{2}{|l|}{$\begin{array}{l}\text { Physical activity and sedentary behaviour } \\
\text { (method of data collection) }\end{array}$} \\
\hline Walkability index (GIS) & 1 & $\begin{array}{l}\text { Weekly frequency of within-neighbourhood and total } \\
\text { walking for transport (S) }\end{array}$ & 1 and 2 \\
\hline $\begin{array}{l}\text { Percentage of older adults in the } \\
\text { neighbourhood (GIS) }\end{array}$ & 1 & $\begin{array}{l}\text { Weekly minutes of within-neighbourhood and total } \\
\text { walking for transport (S) }\end{array}$ & 1 and 2 \\
\hline $\begin{array}{l}\text { Access to recreational facilities and } \\
\text { parks }(G I S+E A+S)\end{array}$ & 1 & $\begin{array}{l}\text { Weekly frequency of within-neighbourhood and total } \\
\text { walking for recreation }(S)\end{array}$ & 1 and 2 \\
\hline Features of public open spaces (EA) & 1 & $\begin{array}{l}\text { Weekly minutes of within-neighbourhood and total } \\
\text { walking for recreation (S) }\end{array}$ & 1 and 2 \\
\hline Access to transit points $(\mathrm{GIS}+\mathrm{AE}+\mathrm{S})$ & 1 & $\begin{array}{l}\text { Weekly minutes of non-walking } \\
\text { moderate-to-vigorous PA (S) }\end{array}$ & 1 and 2 \\
\hline $\begin{array}{l}\text { Access to various destinations and } \\
\text { services }(A E+S)\end{array}$ & 1 & Daily minutes of sitting (S) & 1 and 2 \\
\hline Traffic safety $(A E+S)$ & 1 & Weekly minutes of moderate-to-vigorous $\mathrm{PA}(\mathrm{O})$ & 1 \\
\hline Pedestrian infrastructure $(A E+S)$ & 1 & Weekly minutes of light-to-vigorous PA (O) & 1 \\
\hline Personal safety $(\mathrm{AE}+\mathrm{S})$ & 1 & Daily minutes of sedentary time $(\mathrm{O})$ & 1 \\
\hline Aesthetics $(A E+S)$ & 1 & \multicolumn{2}{|c|}{ Quality of life and depressive symptoms (method of data collection) } \\
\hline Weather (PD) & 1 and 2 & Score on Geriatric Depression Scale (S) & 1 and 2 \\
\hline \multirow[t]{2}{*}{ Air pollution (PD) } & 1 and 2 & Total quality of life score (S) & 1 and 2 \\
\hline & & Quality of life domain score (S) & 1 and 2 \\
\hline \multicolumn{2}{|l|}{$\begin{array}{l}\text { Covariates, moderators and/or } \\
\text { mediators }\end{array}$} & \multicolumn{2}{|l|}{ Covariates, moderators and/or mediators } \\
\hline \multicolumn{2}{|c|}{ Sociodemographics (method of data collection) } & \multicolumn{2}{|l|}{ Health indicators (method of data collection) } \\
\hline Age (S) & 1 & Body mass index $(\mathrm{O})$ & 1 \\
\hline Sex (S) & 1 & Health status $(\mathrm{O}+\mathrm{S})$ & 1 and 2 \\
\hline Educational attainment (S) & 1 & Instrumental activities of daily living (S) & 1 \\
\hline Marital status (S) & 1 & Functional mobility $(\mathrm{O})$ & \\
\hline Housing (S) & 1 & Perceived home environment & \\
\hline Living arrangements (S) & 1 & Perceived building quality (S) & 1 \\
\hline $\begin{array}{l}\text { Neighbourhood-level median } \\
\text { household income (GIS) }\end{array}$ & 1 & Travel behaviour & \\
\hline Car ownership in household (S) & 1 & $\begin{array}{l}\text { Frequency and mode of transport to commonly } \\
\text { visited destinations (S) }\end{array}$ & 2 \\
\hline \multicolumn{2}{|c|}{ Psychosocial factors (method of data collection) } & \multicolumn{2}{|l|}{ Psychosocial factors (method of data collection) } \\
\hline Social networks (S) & 2 & Self-efficacy for PA (S) & 1 \\
\hline Barriers to PA (S) & 1 & Neighbourhood satisfaction (S) & 2 \\
\hline Social support for PA (S) & 1 & & \\
\hline
\end{tabular}

All public open spaces intersecting participants' residential buffers are audited. As it has been shown that a sample of $50 \%$ of street segments within a neighbourhood can provide estimates of various environmental features representative of a neighbourhood, ${ }^{62}$ only half of the segments within each residential buffer are assessed.

\section{Perceived neighbourhood environment}

It remains unclear whether objective measures of the neighbourhood environment are more potent correlates of PA and sedentary time than perceived measures. Cerin et $a t^{42}$ developed and validated the Neighbourhood Environment Walkability Scale for Chinese Seniors (NEWS-CS) to assess the perceived aspect of the neighbourhood environment in Hong Kong. This scale assesses perceived features that conceptually overlap with those assessed by the EAST-HK. These include: perceived access to recreational facilities and parks, personal safety (including social environment variables, such as the presence of people on the street and crime), traffic safety, access to transit points, aesthetics, pedestrian infrastructure, and access to a variety of destinations. The NEWS-CS has been shown to be a reliable and valid measure of perceived neighbourhood characteristics associated with walking and other types of PA in older adults. ${ }^{11} 1255$

\section{Climate and air pollution}

Weather is an environmental factor that is not well studied in the context of Hong Kong older adults' PA and QoL. However, there is some evidence that it impacts PA in 
Hong Kong dwellers. ${ }^{64}$ Daily weather data, which are publicly available from the Hong Kong Observatory, are being recorded throughout the entire participant data collection period. Variables collected include high and low temperature, humidity and precipitation. Information on concentrations of air pollutants (respirable suspended particulates; gaseous pollutants; toxic air pollutants) is obtained from the Environmental Protection Department of Hong Kong. It is expected that humid and hot weather conditions and air pollution will be negatively associated with PA and QoL.

\section{Outcomes}

\section{$P A$ and $S B$}

The Chinese version of the International Physical Activity Questionnaire (IPAQ-C) - Short (past 7 days) is used to measure self-reported weekly minutes of moderateto-vigorous PA other than walking and daily minutes of sitting. The IPAQ-C has been shown to have good reliability and validity in Chinese older adults. ${ }^{65}$ Weekly frequency and minutes of within-neighbourhood and outside-neighbourhood walking for transportation and recreation are assessed using the Neighbourhood Walking Questionnaire for Chinese Seniors (NWQ-CS), ${ }^{66}$ which represents a validated version of the walking items of the Neighbourhood Physical Activity Questionnaire ${ }^{67}$ adapted for Cantonese-speaking older adults.

Weekly and daily minutes of moderate-to-vigorous PA, light-to-vigorous PA and daily minutes of sedentary time are objectively assessed in a study subsample using the triaxial accelerometer Actigraph GT3X for seven consecutive days. The Actigraph is a small device worn on an elastic belt that provides an objective measure of PA and sedentary time. The participants are instructed to firmly secure it to the right hip in the mid-axillary line, wear it during waking hours, remove it for water activities and sleep, and keep a concurrent log to record the periods of monitor wearing and non-wearing. Non-wear periods are identified using the $\log$ information and recordings of $100 \mathrm{~min}$ of consecutive zero counts, a criterion appropriate for an older population. ${ }^{68}{ }^{69}$ A valid day is defined as having at least $10 \mathrm{~h}$ of recorded activity and valid data are considered five valid days, including at least one weekend day. Previously published cutpoints, which have been employed in earlier studies with older adults, ${ }^{6869}$ are used to classify activity counts into sedentary $(<100$ counts/min), light (100-1951 counts/ $\mathrm{min})$, moderate (1952-5724 counts/min) and vigorous (>5724 counts $/ \mathrm{min}) .{ }^{70}$ Accelerometer data integrity is monitored, and in cases of unusable or missing data participants are asked to re-wear the Actigraph for the required number of days.

\section{Quality of life}

Quality of life is assessed using the extensively validated Hong Kong Chinese WHO Quality of Life Scale-Brief Version appropriate for use with older adults. ${ }^{72}$ This version includes 28 items, covering four domains: psychological health, physical health, social relationship and the environment. The scales yield a score for each domain and a total QoL score.

\section{Depressive symptoms}

Depressive symptoms are measured using the Chinese version of the four-item Geriatric Depression Scale (GDS). ${ }^{73}$ Versions of the GDS are the most widely used scales for the detection of depression in older adults. They have good sensitivity and specificity. ${ }^{74}$ Participants with a score of 2 or higher are considered to be potentially depressed.

\section{Covariates, moderators and/or mediators \\ Perceived home environment}

Neighbourhood-level environmental variables are the main focus of this study. However, the home environment (quality of residential building) can potentially impact on QoL ${ }^{75}$ and the likelihood of spending time outdoors. The Building Environment Quality Questionnaire ${ }^{75}$ is used to assess perceived air quality, water quality, sanitation, space, light, sound and thermal comfort in residential buildings. Scores on these domains can be added up to yield a total quality of building score. A study conducted on 350 adult residents of Hong Kong has provided support for the reliability and validity of the scale. ${ }^{75} \mathrm{~A}$ total quality of building score will be used as a covariate.

\section{Sociodemographics}

Data on age, sex, educational attainment, car ownership in the household and other sociodemographic variables listed in table 1 are collected from the participants. These variables will serve as covariates and moderators in the main analyses. For example, we will examine whether associations of neighbourhood environmental attributes with PA vary by levels of educational attainment and by sex. As noted above, neighbourhood-level socioeconomic status is assessed using GIS and Census data on median household income for each participant's residential buffer.

\section{Health indicators}

Physical health and the presence of chronic diseases are assessed using information from clinical health problems checklists obtained from the EHCs (based on medical staff assessments) or the participant, if these are not available. The validated Chinese version of the eightitem Instrumental Activities of Daily Living (IADL) is used to assess the ability of older people to perform their IADLs. ${ }^{76}$ Higher scores indicate better functioning. The height and weight of each participant is measured by medical staff or trained field researchers using a stadiometer and calibrated scales. BMI will be calculated as weight divided by squared height. Functional mobility is objectively assessed using the Short Physical Performance Battery, ${ }^{77}$ which includes tests of side-by-side, semi-tandem and tandem standing for at 
least $10 \mathrm{~s}$, walking speed over $3 \mathrm{~m}$, and time to complete five chair rises. These variables will be used as covariates and moderators, depending on the research question.

\section{Travel behaviour}

Participants are asked to report the common destinations they visit for recreation, shopping or other errands and eating/food-purchase purposes; the frequency with which they visit them; the modes of transport they use to get to/from these destinations; and the amount of time it takes to get to/from these destinations. Similar questions were used in an earlier study on Hong Kong older adults. ${ }^{78}$ These variables will be examined as mediators of environment-QoL and depressive symptoms associations. It is postulated that the environment will in part determine the accessibility of destinations important for daily living, and the ease of access of these destinations will be associated with QoL and depressive symptoms.

\section{Psychosocial factors}

Psychosocial variables associated with PA in older adults are also assessed. These include self-efficacy for leisure time PA and transport-related walking; perceived barriers to engagement in leisure time PA and transport-related walking; and family and friends' support for engagement in leisure time PA and transport-related walking. These scales have been validated in previous studies, including older Chinese. ${ }^{7}$ They will be examined as covariates, moderators or mediators, depending on the research question and objective (see study aims above). The inclusion of these variables will allow the examination of a true multilevel socioecological model of PA and QoL by accounting for and separating the various layers of influence (ie, individual, social and physical environments). ${ }^{26}$ Finally, participants are also asked to provide data on social networks (accessibility, composition and size). ${ }^{43}$ Similar to the other psychosocial factors, these will be modelled as moderators or mediators, depending on the research question and study objective.

\section{Procedures}

On recruitment, verification of eligibility, and provision of written informed consent to take part in the study, trained research assistants schedule a one-to-one interview with the participants. All surveys are intervieweradministered. The first and longer assessment involves a maximal $2.5 \mathrm{~h}$ interview (average $1.5 \mathrm{~h}$ ), including breaks when needed. However, if necessary, the interview is conducted over two separate occasions. The second and shorter interview $(1 \mathrm{~h})$ is scheduled approximately 6 months after the first interview. On entering the study, all participants are asked whether they would be willing to wear the accelerometer for a week. Approximately $45 \%$ of the participants are randomly chosen for this component of the study. The participants taking part in the accelerometry component of the study need to be literate, that is, be able to keep a log. The project phone number is provided in case of questions during the week of monitoring. These participants receive two daily phone calls at predetermined times to motivate participation and verify compliance. Other reminders/ prompts are provided, including a study pamphlet to be put on the inside of their home entrance door or at any other appropriate location where it can be easily seen. A modest incentive $(\mathrm{HK} \$ 50)$ and participation certificate are provided for completing the baseline and 6-month interviewer-administered surveys. Participants taking part in the 7-day accelerometer component (approximately $45 \%$ of the sample) receive an additional incentive of HK\$100. All participants enter a draw to win one of three gift certificates valued at $\mathrm{HK}$ $\$ 2000$. Incentives are meant to help increase recruitment and retention rates. Every effort is made to retain participants, based on our and others' experience. Methods include motivational phone calls and brief newsletters about the study progress. On the basis of previous experience, we estimate an $80+\%$ retention rate across the two assessments.

\section{Data analyses}

The proposed data set will have a hierarchical structure consisting of person-level observations nested within neighbourhoods (TPUs). The main aims of the study are to examine confounder-adjusted associations of environmental variables with $\mathrm{PA}, \mathrm{QoL}$ and depressive symptoms; and moderators and mediators of these associations. For this purpose, generalised additive mixed models (GAMMs) with random intercepts will be used. These can account for multiple sources of dependency (neighbourhoods and multiple assessments on the same participants), different types of data (eg, continuous or binary) following normal or other types of distributions (eg, negative binomial, gamma), and model curvilinear relationships of complex form. ${ }^{79-81}$ They can also handle missing data at multiple assessments. Curvilinear relationships will be depicted in graphical form. ${ }^{80}$ GAMMs perform well when the number of observations across areas is unbalanced, ${ }^{82}$ which may be relevant to this study as the number of participants may vary substantially across TPUs. Moderators of associations will be identified by adding appropriate interaction terms to the relevant GAMMs. Significant interaction effects will be probed by estimating associations at meaningful values of the moderator. Mediation effects will be evaluated using the joint significance test ${ }^{83}$ whereby associations of exposure measures (eg, neighbourhood environmental attributes) with potential mediators (eg, PA) and exposure-measure-adjusted associations of mediators with outcomes (eg, QoL) will be estimated. A probability level of 0.05 will be adopted for all analyses.

\section{ETHICS AND DISSEMINATION}

On recruitment, participants are given detailed verbal and written information on the study and, after their eligibility is assessed, they are requested to provide written 
informed consent before the first one-to-one interview is scheduled for data collection purposes. They are informed that they can withdraw from the study at any time without prejudice. On entering the study, each participant is assigned a unique study code. Data from interviews are entered on hard copies of the questionnaire that can be linked to specific participants using the study codes assigned to them. These study codes are kept separately from any participants' personal identifiers (name, address and contact details) and are accessible only to qualified research staff and team members and saved on a password-secured server. Only the named researchers have access to the data. Data will be stored for 10 years and then permanently destroyed.

The experience of discomfort or embarrassment during data collection is an unlikely event. There are no known health risks or problems associated with wearing accelerometers. There may be some embarrassment associated with wearing the device, but these are minimal risks. Participants are given belts on which to wear the devices under clothing to minimise potential embarrassment. With regard to the interviews, the questions asked are not particularly sensitive. Also, the participants are informed that they may refuse to answer any question they may consider sensitive without incurring any negative consequences. The physical performance test we conduct was especially devised for older adults and poses no health risks. Height and weight measurements are taken in a private office/room with gender appropriate research officers. All data are kept private and confidential.

\section{Dissemination plan}

It is anticipated that the findings from this study will be of interest to a diverse audience, including public health professionals, national and provincial urban planning associations, clinicians and members of the public. They will also appeal to national and international researchers due to the innovativeness of the research questions tackled and the multimethod approach employed to gather data on the neighbourhood environment and PA.

Findings are being and will be disseminated via various channels. Participants receive a report detailing their individual results. We engage with the Department of Health of Hong Kong and national and international urban planning interest groups to provide updates on novel findings arising from the study that are relevant to health and urban planning policies and practices. The Department of Geography of the University of Hong Kong will be hosting an international conference in June 2016, where we intend to provide an overview of the main results. The same will be done at a conference on active ageing in Melbourne (Australia) in July 2016. Members of the research team are leading and/or co-organising these two conferences. We intend to disseminate study findings through high-calibre international medical, psychology, urban planning and/or public health peer-reviewed journals. Importantly, we plan to present and discuss the results of our study with relevant stakeholders, such as the EHCs, the Department of Health of Hong Kong SAR, urban planning associations and elderly community centres. We will also use our informal networks to disseminate our findings nationally and internationally.

Author affiliations

${ }^{1}$ School of Public Health, The University of Hong Kong, Hong Kong, Hong Kong SAR, China

${ }^{2}$ Centre for Physical Activity and Nutrition Research, School of Exercise and Nutrition Sciences, Deakin University, Burwood, Victoria, Australia

${ }^{3}$ Department of Sports Science and Physical Education, Faculty of Education, The Chinese University of Hong Kong, Hong Kong, Hong Kong SAR, China

${ }^{4}$ Institute for Health \& Ageing, Australian Catholic University, Melbourne, Victoria, Australia

${ }^{5}$ Elderly Health Service, Department of Health, The Government of Hong Kong Special Administrative Region, Hong Kong, China

${ }^{6}$ Department of Geography, Faculty of Social Sciences, The University of Hong Kong, Hong Kong, Hong Kong SAR, China

Contributors EC drafted the manuscript. CHPS, AB, MMCC, PL and RSYL contributed to the conception and the design of the study. JMJ and CJPZ contributed to the conceptualisation of the manuscript. All authors provided feedback during manuscript development. All the authors read and approved the final manuscript.

Funding This study received a General Research Fund grant from the University Grant Committee (Hong Kong) (HKU 741511H). EC is supported by an ARC Future Fellowship FT 140100085.

Disclaimer The information reported in this paper are those of the authors and are independent from the funding sources.

Competing interests None declared.

Ethics approval Ethical approval was received by the University of Hong Kong Human Research Ethics Committee for Non-Clinical Faculties (EA270211) and the Department of Health (Hong Kong SAR).

Provenance and peer review Not commissioned; peer reviewed for ethical and funding approval prior to submission.

Data sharing statement No additional data are available.

Open Access This is an Open Access article distributed in accordance with the Creative Commons Attribution Non Commercial (CC BY-NC 4.0) license, which permits others to distribute, remix, adapt, build upon this work noncommercially, and license their derivative works on different terms, provided the original work is properly cited and the use is non-commercial. See: http:// creativecommons.org/licenses/by-nc/4.0/

\section{REFERENCES}

1. Paterson DH, Jones GR, Rice CL. Aging and physical activity: evidence to develop exercise recommendations for older adults. Can J Public Health 2007;98(Suppl 2):S69-108.

2. Department of Health, UK. Physical activity, health improvement and prevention: at least five a week. London, UK: Department of Health, 2004.

3. Vogel T, Brechat PH, Leprêtre PM, et al. Health benefits of physical activity in older patients: a review. Int J Clin Pract 2009;63:303-20.

4. Yorston LC, Kolt GS, Rosenkranz RR. Physical activity and physical functioning older adults: the 45 and Up Study. J Am Geriatr Soc 2012;60:719-25.

5. Buman MP, Hekler EB, Haskell WL, et al. Objective light-intensity physical activity associations with rated health in older adults. $\mathrm{Am} \mathrm{J}$ Epidemiol 2010;172:1156-65.

6. de Rezende LF, Rey-López JP, Matsudo VK, et al. Sedentary behavior and health outcomes among older adults: a systematic review. BMC Public Health 2014;14:333.

7. Cheng $\mathrm{YH}$, Chou $\mathrm{KL}$, Macfarlane DJ, et al. Identifying the pattern of physical exercise and determinants contributing to the engagement 
and exercise among Hong Kong older adults. Final report HSRF/ NCPF \#213025 submitted to the Grant Review Board. Hong Kong: HKSAR, 2004.

8. Sun F, Norman IJ, While AE. Physical activity in older people: a systematic review. BMC Public Health 2013;13:449.

9. Bauman AE, Reis RS, Sallis JF, et al. Correlates of physical activity: why are some people physically active and others not? Lancet 2012;380:258-71.

10. Van Dyck D, Cerin E, Conway TL, et al. Associations between perceived neighborhood environmental attributes and adults' sedentary behavior: findings from the U.S.A, Australia and Belgium. Soc Sci Med 2012;74:1375-84.

11. Cerin E, Macfarlane D, Sit HP, et al. Effects of the built environment on walking among Hong Kong older adults. Hong Kong Med $J$ 2013;19:39-41.

12. Cerin $\mathrm{E}$, Sit $\mathrm{CH}$, Barnett $\mathrm{A}$, et al. Walking for recreation and perceptions of the neighborhood environment in older Chinese urban dwellers. J Urban Health 2013:90:56-66.

13. Van Cauwenberg J, De Bourdeaudhuij I, De Meester F, et al. Relationship between the physical environment and physical activity in older adults: a systematic review. Health Place 2011;17:458-69.

14. Van Holle V, Van Cauwenberg J, Van Dyck D, et al. Relationship between neighborhood walkability and older adults' physical activity: results from the Belgian Environmental Physical Activity Study in Seniors (BEPAS Seniors). Int J Behav Nutr Phys Act 2014; $11: 110$.

15. United Nations, Department of Economic and Social Affairs. Political declaration and Madrid international plan of action on ageing. New York: United Nations, 2002.

16. Cerin $\mathrm{E}$, Lee KY, Barnett $\mathrm{A}$, et al. Walking for transportation in Hong Kong Chinese urban elders: a cross-sectional study on what destinations matter and when. Int J Behav Nutr Phys Act 2013;10:78

17. Barnett A, Cerin E, Ching C, et al. Neighbourhood environment sitting time and motorised transport in older adults: a cross-sectional study in Hong Kong. BMJ Open 2015;5:e007557.

18. Cerin $E$, Lee KY, Barnett $A$, et al. Objectively-measured neighborhood environments and leisure-time physical activity in Chinese urban elders. Prev Med 2013;56:86-9.

19. Lockett D, Willis A, Edwards N. Through seniors' eyes: an exploratory qualitative study to identify environmental barriers to and facilitators of walking. Can J Nurs Res 2005;37:48-65.

20. Satariano WA, Ivey SI, Kurtovich E, et al. Lower-body function, neighborhoods, and walking in an older population. Am J Prev Med 2010;38:419-28.

21. Sims J, Hill K, Davidson S, et al. A snapshot of the prevalence of physical activity amongst older, community dwelling people in Victoria, Australia: patterns across the 'young-old' and 'old-old'. BMC Geriatr 2007;7:4.

22. Cerin E, Mellecker R, Macfarlane DJ, et al. Socio-economic status, neighbourhood characteristics, and walking within the neighbourhood among older Hong Kong Chinese. J Aging Health 2013;28:1425-44.

23. Michael YL, Perdue LA, Orwoll ES, et al. Physical activity resources and changes in walking in a cohort of older men. Am J Public Health 2010;1000:654-60.

24. Van Cauwenberg J, Cerin E, Timperio A, et al. Park proximity, quality and recreational physical activity among mid-older aged adults: moderating effects of individual factors and area of residence. Int J Behav Nutr Phys Act 2015;12:46.

25. Nagel CL, Carlson NE, Bosworth M, et al. The relation between neigbourhood built environment and walking activity among older adults. Am J Epidemiol 2008;168:461-8.

26. Sallis JF, Owen N. Physical activity and behavioural medicine. Thousands Oaks, CA: Sage, 1999.

27. Van Holle V, Van Cauwenberg J, Deforche B, et al. Do psychosocia factors moderate the association between objective neighborhood walkability and older adults' physical activity? Health Place 2015;34:118-25.

28. Coffee NT, Howard N, Paquet C, et al. Is walkability associated with a lower cardiometabolic risk? Health Place 2013;21:163-9.

29. Li F, Hamer P, Cardinal BJ, et al. Built environment and changes in blood pressure in middle aged and older adults. Prev Med 2009;48:237-41.

30. Parra DC, Gomez LF, Sarmiento OL, et al. Perceived and objective neighbourhood environment attributes and health related quality of life among the elderly in Bogotá, Colombia. Soc Sci Med 2010;70:1070-6.

31. Roswall N, Høgh V, Envold-Bidstrup P, et al. Residential exposure to traffic noise and health-related quality of life-a population-based study. PLOS ONE 2015;10:e120199.
32. Saarloos D, Alfonso H, Giles-Corti B, et al. The built environment and depression in later life: the health in men study. Am J Geriatr Psychiatry 2011;19:461-70.

33. Kaczynski AT, Potwarka LR, Saelens BE. Associations of park size, distance, and features with physical activity in neighbourhood parks. Am J Public Health 2008;98:1451-6.

34. Richard L, Gauvin L, Gosselin C, et al. Staying connected: neighbourhood correlates of social participation among older adults living in an urban environment in Montreal, Quebec. Health Prom Int 2008;24:46-57.

35. White DK, Jette AM, Felson DT, et al. Are features of the neighborhood environment associated with disability in older adults? Disabil Rehabil 2010;32:639-45.

36. Leslie E, Cerin E. Are perceptions of the local environment related to neighbourhood satisfaction and mental health in adults? Prev Med 2008;47:273-8.

37. du Toit L, Cerin E, Leslie E, et al. Does walking in the neighbourhood enhance local sociability? Urban Studies 2007;44:1677-95

38. Buffel T, De Donder L, Phillipson C, et al. Social participation among older adults living in medium-sized cities in Belgium: the role of neighbourhood perceptions. Health Promot Int 2014;29:655-68.

39. King K. Neighborhood walkable urban form and C-reactive protein. Prev Med 2013;57:850-4.

40. Frei $P$, Mohler E, Röösli M. Effect of nocturnal road traffic noise exposure and annoyance on objective and subjective sleep quality. Int J Hyg Environ Health 2014;217:188-95.

41. Cerin E, Macfarlane D, Ko HH, et al. Measuring perceived neighbourhood walkability in Hong Kong. Cities 2007;24:209-17.

42. Cerin E, Sit $\mathrm{CH}$, Cheung MC, et al. Reliable and valid NEWS for Chinese seniors: measuring perceived neighborhood attributes related to walking. Int J Behav Nutr Phys Act 2010;7:84.

43. Cheng ST, Lee CK, Chan AC, et al. Social network types and subjective well-being in Chinese older adults. J Gerontol B Psychol Sci 2009;64:713-22.

44. Prins RG, van Lenthe FJ. The hour-to-hour influence of weather conditions on walking and cycling among Dutch older adults. Age Ageing 2015;44:886-90.

45. Alahmari AD, Mackay AJ, Patel AR, et al. Influence of weather and atmospheric pollution on physical activity in patients with COPD. Respir Res 2015;16:71.

46. Hunter DJ, Riordan EA. The impact of arthritis on pain and quality of life: an Australian survey. Int $J$ Rheum Dis 2014;17:149-55.

47. Cerin E, Barnett A, Cheung MC, et al. Reliability and validity of the IPAQ-L in a sample of Hong Kong urban older adults: does neighborhood of residence matter? J Aging Phys Act 2012;20:402-20.

48. Eves FF, Masters RS, McManus A, et al. Contextual barriers to lifestyle physical activity interventions in Hong Kong. Med Sci Sports Exerc 2008;40:965-71.

49. Merrill RM, Shields EC, White GL Jr, et al. Climate conditions and physical activity in the United States. Am J Health Behav 2005;29:371-81.

50. Vandenbroucke JP, Von Elm E, Altman DG, et al. Strengthening the Reporting of Observational Studies in Epidemiology (STROBE): explanation and elaboration. PLoS Med 2007:4:e297.

51. Owen N, Cerin E, Leslie E, et al. Neighborhood walkability and the walking behavior of Australian adults. Am J Prev Med 2007:33:387-95.

52. Cerin E, Leslie E, Owen N, et al. An Australian version of the neighborhood environment walkability scale: construct and factorial validity. Meas Phys Educ Exerc Sci 2008;12:31-51.

53. Leslie E, Coffee N, Frank L, et al. Walkability of local communities: using Geographic Information Systems to objectively assess relevan environmental attributes. Health Place 2007;13:111-22.

54. Frank L, Kerr J, Rosenberg D, et al. Healthy aging and where you live: community design relationships with physical activity and body weight in older Americans. J Phys Act Health 2010;7:S82-90.

55. Cerin $\mathrm{E}$, Sit $\mathrm{CH}$, Barnett $\mathrm{A}$, et al. Aging in an ultra-dense metropolis: perceived neighbourhood characteristics and utilitarian walking in Hong Kong elders. Public Health Nutr 2014;17:225-32.

56. Hong Kong Government-Personal Data Ordinance. 2015. https:// www.pcpd.org.hk/english/data_privacy_law/ordinance_at_a_Glance/ ordinance.html

57. Schooling CM, Lam TH, Li ZB, et al. Obesity, physical activity, and mortality in a prospective Chinese elderly cohort. Arch Intern Med 2006;166:1498-504.

58. Chiu HF, Lam LC, Chi I, et al. Prevalence of dementia in Chinese elderly in Hong Kong. Neurology 1998;50:1002-9.

59. Adams MA, Frank LD, Schiperijn J, et al. International variation in neighborhood walkability transit and recreation environments using 
Geographic information systems: the IPEN Adult study. Int $\mathrm{J}$ Health Geographics 2014;13:14

60. Cerin E, Conway TL, Cain KL, et al. Sharing good NEWS across the world: developing comparable scores across 12 countries for the neighborhood environment walkability scale (NEWS). BMC Public Health 2013;13:309.

61. Li F, Fisher KJ, Brownson RC. A multilevel analysis of change in neighbourhood walking activity in older adults. J Aging Phys Act 2005;13:145-59.

62. Cerin E, Chan KW, Macfarlane DJ, et al. Objective assessment of walking environments in ultra-dense cities: development and reliability of the environment in Asia Scan Tool-Hong Kong version (EAST-HK). Health Place 2011;17:937-45.

63. Broomhall M, Giles-Corti B, Lange A. Quality of Public Open Space Tool (POST). Perth, WA: School of Population Health, The University of Western Australia, 2004.

64. Eves FF, Masters RS, McManus AM. Effects of point-of-choice stair climbing interventions in Hong Kong. Hong Kong Med J 2008;14(5 Suppl):36-9.

65. Deng HB, Macfarlane DJ, Thomas GN, et al. Reliability and validity of the IPAQ-Chinese: the Guangzhou Biobank Cohort study. Med Sci Sports Exerc 2008;40:303-7.

66. Cerin $\mathrm{E}$, Barnett $\mathrm{A}$, Sit $\mathrm{CH}$, et al. Measuring walking within and outside the neighborhood in Chinese elders: reliability and validity. BMC Public Health 2011;11:851.

67. Giles-Corti B, Timperio A, Cutt $\mathrm{H}$, et al. Development of a reliable measure of walking within and outside the local neighborhood: RESIDE's neighborhood physical activity questionnaire. Prev Med 2006:42:455-9.

68. Davis MG, Fox KR. Physical activity patterns assessed by accelerometry in older people. Eur J Appl Phys 2007;100:581-9.

69. Davis MG, Fox KR, Hillsdon M, et al. Objectively measured physical activity in a diverse sample of older urban UK adults. Med Sci Sports Exerc 2011:43:647-54.

70. Craig CL, Marshall AL, Sjöström M, et al. International physical activity questionnaire: 12 -country reliability and validity. Med $\mathrm{SCl}$ Sports Exerc 2003;35:1381-95.

71. Freedson PS, Melanson E, Sirard J. Calibration of the computer science and applications, Inc. accelerometer. Med Sci Sports Exerc 1998;30:777-81.
72. Leung KF, Tay M, Cheng SS, et al. Hong Kong Chinese version World Health Organization Quality of Life Measure-Abbreviated Version WHOQoL-BREF(HK). Hong Kong, HKSAR: Hong Kong Hospital Authority, 1997.

73. Cheng ST, Chan AC. Comparative performance of long and short forms of the geriatric depression scale in mildly demented Chinese. Int J Geriatr Psychiatry 2005;20:1131-7.

74. Cheng ST, Chan AC. Validity of the GDS-4 revisited. Psychol Health Med 2008;13:621-6.

75. Chan KW, MacFarlane DJ, Cerin E, et al. Reliability and validity of a new building environmental quality questionnaire. Indoor Built Environ 2010;19:599-611.

76. Lawton MP, Brody EM. Assessment of older people: self-maintaining and instrumental activities of daily living. Gerontologist 1969:9:179-86.

77. Guralnik JM, Simonsick EM, Ferrucci L, et al. A short physical performance battery assessing lower extremity function: association with self-reported disability and prediction of mortality and nursing home admission. J Gerontol 1994; 49:M85-94.

78. Barnett A, Cerin E, Cheung MC, et al. An in-depth pilot study on patterns, destinations, and purposes of walking in Hong Kong older adults. J Aging Phys Act 2015;23:144-52.

79. Cerin E. Statistical approaches to testing the relationships of the built environment with resident-level physical activity behavior and health outcomes in cross-sectional studies with cluster sampling. J Plann Lit 2011;26:151-67.

80. Cerin E, Cain K, Conway TL, et al. Neighborhood environments and objectively measured physical activity in 11 countries. Med Sci Sports Exerc 2014;44:2253-64.

81. Wood SN. Generalized additive models: an introduction with $R$ Boca Raton, FL: Chapman \& Hall, 2006.

82. Burton P, Gurrin L, Sly P. Extending the simple linear regression model to account for correlated responses: an introduction to generalized estimating equations and multi-level mixed modeling. Stat Med 1998;17:1261-91.

83. Cerin E, Taylor L, Leslie E, et al. Small-scale randomized controlled trials need more powerful methods of mediational analysis than Baron and Kenny's. J Clinical Epidemiol 2006;59:457-64. 